\title{
Indications of nonlinear deterministic and finite-dimensional structures in time series of brain electrical activity: Dependence on recording region and brain state
}

\author{
Ralph G. Andrzejak, ${ }^{1,2, *}$ Klaus Lehnertz, ${ }^{1, \dagger}$ Florian Mormann, ${ }^{1,2}$ Christoph Rieke, ${ }^{1,2}$ Peter David, ${ }^{2}$ and Christian E. Elger ${ }^{1}$ \\ ${ }^{1}$ Department of Epileptology, University of Bonn, Sigmund-Freud-Strasse 25, 53105 Bonn, Germany \\ ${ }^{2}$ Institut für Strahlen- und Kernphysik, University of Bonn, Nußallee 14-16, 53115 Bonn, Germany
}

(Received 14 May 2001; published 20 November 2001)

\begin{abstract}
We compare dynamical properties of brain electrical activity from different recording regions and from different physiological and pathological brain states. Using the nonlinear prediction error and an estimate of an effective correlation dimension in combination with the method of iterative amplitude adjusted surrogate data, we analyze sets of electroencephalographic (EEG) time series: surface EEG recordings from healthy volunteers with eyes closed and eyes open, and intracranial EEG recordings from epilepsy patients during the seizure free interval from within and from outside the seizure generating area as well as intracranial EEG recordings of epileptic seizures. As a preanalysis step an inclusion criterion of weak stationarity was applied. Surface EEG recordings with eyes open were compatible with the surrogates' null hypothesis of a Gaussian linear stochastic process. Strongest indications of nonlinear deterministic dynamics were found for seizure activity. Results of the other sets were found to be inbetween these two extremes.
\end{abstract}

DOI: 10.1103/PhysRevE.64.061907

PACS number(s): 87.19.La, 05.45.-a, 05.45.Tp, 87.19.Xx

\section{INTRODUCTION}

The theory of deterministic chaos deals with complex dynamical systems that are characterized by the fact that they can be rather simple to describe, e.g., by a set of nonlinear differential equations, but can show a complicated, often erratic, temporal evolution [1]. Nonlinearity as a necessary condition for such chaotic behavior is present in many dynamical systems found in nature. For a neuronal network such as the brain, nonlinearity is introduced even on the cellular level, since the dynamical behavior of individual neurons is governed by threshold and saturation phenomena. Moreover, the hypothesis of an entirely stochastic brain can be rejected due to its ability to perform sophisticated cognitive tasks. For these reasons, the electroencephalogram (EEG) appears to be an appropriate area for nonlinear time series analysis (NTSA) techniques, the practical spin-off from the theory of deterministic chaos [2].

The structure of the brain, however, is highly complicated. Furthermore, the EEG always results from a huge number of individual neurons, each interacting with its neighboring neurons as well as with remote neurons whose electric potentials are not included in the measurement. It is therefore questionable whether EEG time series, particularly those of short duration, can carry enough information to reveal dynamical properties of the underlying system brain. Many studies are known from the literature in which NTSA techniques were applied to different kinds of EEGs from humans, such as recordings from healthy volunteers at rest [3-10], sleep [11-13], during periods of cognitive activity [14-17], under the influence of low doses of ethanol [18] or anesthetics [19], or from patients with diseases like Alzheimer's [16,20], Parkinson's [21,22], Creutzfeldt-Jakob

\footnotetext{
*Electronic address: ralphandrzejak@yahoo.de

†Electronic address: klaus.lehnertz@ukb.uni-bonn.de
}

[23], depression [24], and schizophrenia [25] mostly during cognitive activity and in comparison against healthy control subjects. In particular, recordings from epilepsy patients have often attracted researchers' attention [9,26-41]. This is due to outstanding features of actual seizure activity and, moreover, to the medical indication to perform recordings invasively in epilepsy patients, which offers a unique view of the dynamical system human brain.

Interpretations of results ranged from "evidences for chaotic attractors" underlying the alpha rhythm [7], sleep recordings [11], or epileptic seizures $[33,35,40]$, to the conclusion that EEG data of healthy volunteers "may be more appropriately modeled by linearly filtered noise" [15]. In between these two extremes, authors concluded significant indications of nonlinearity but no indication of low dimensionality or determinism $[3-5,8,13]$. Besides the aim of finding a certain dynamical model for the EEG, it was furthermore investigated if relative changes of the calculated quantities are capable of differentiating between different physiological brain states $[14,15,17]$, increasing insight into brain dysfunction $[9,16,21-25]$ or even of yielding information useful for diagnostic purposes [26,28-30,34]. The anticipation of impending epileptic seizures is a further challenging aspect that was investigated by a number of studies [31,36-39].

Nevertheless, it remains uncertain whether the aforementioned varying results indeed reflect different dynamical features of brain electrical activity, or whether they must instead be attributed to differences in parameters of the respective algorithms and recording setups. Thus the aim of our study is to compare dynamical properties of brain electrical activity from different extracranial and intracranial recording regions and from different physiological and pathological brain states, using fixed analysis parameters. Apart from the different recording electrodes used for extracranial and intracranial EEG registration, all other recording parameters were fixed. Some of the morphological characteristics of the different EEG time series under investigation, which are obvious to an expert's eye, will be sketched in the following. 
EEG time series recorded extracranially during the relaxed state of healthy subjects with eyes closed show a predominant physiological rhythm, the so-called alpha rhythm in a frequency range of $8-13 \mathrm{~Hz}$, an activity which is most pronounced at the back of the head [42]. In contrast, broader frequency characteristics are obtained for open eyes. EEG time series are also recorded intracranially in humans, however only in the framework of a presurgical evaluation of focal epilepsies. In this context the implantation of electrodes is carried out to exactly localize the seizure generating area which is termed the epileptogenic zone [43]. During a seizure free interval the EEG recorded from within the epileptogenic zone is often characterized by intermittent occurrences of so-called interictal epileptiform activities. Investigation of these steep, sometimes rhythmic high amplitude patterns in EEG recordings contributes to a localization of the epileptogenic zone. Fewer and less pronounced interictal epileptiform activities can be found at recording sites distant from the epileptogenic zone. Finally, the EEG recorded during epileptic seizures, termed ictal activity, is almost periodic and of high amplitude, resulting from hypersynchronous activity of large assemblies of neurons.

We tested indications of deterministic and/or lowdimensional structures in the aforementioned EEG time series against the null hypothesis that these properties are compatible with a Gaussian linear stochastic and stationary process that was passed through a monotonic static but a possibly nonlinear measurement function. To this end, a nonlinear prediction error [44] and an estimate of the correlation dimension [45] were calculated in a reconstructed state space [46] for both the original EEG time series and an ensemble of surrogate time series [47]. To reduce the probability of rejections of this null hypothesis, due solely to nonstationarity, EEG segments were chosen under an inclusion criterion of weak stationarity.

\section{METHODS}

\section{A. Data selection and recording techniques}

Five sets (denoted A-E) each containing 100 singlechannel EEG segments of 23.6-sec duration, were composed for the study. These segments were selected and cut out from continuous multichannel EEG recordings after visual inspection for artifacts, e.g., due to muscle activity or eye movements. In addition, the segments had to fulfill a stationarity criterion described in detail in Sec. II B. Sets A and B consisted of segments taken from surface EEG recordings that were carried out on five healthy volunteers using a standardized electrode placement scheme (cf. Fig. 1). Volunteers were relaxed in an awake state with eyes open (A) and eyes closed (B), respectively. Sets C, D, and E originated from our EEG archive of presurgical diagnosis. For the present study EEGs from five patients were selected, all of whom had achieved complete seizure control after resection of one of the hippocampal formations, which was therefore correctly diagnosed to be the epileptogenic zone (cf. Fig. 2). Segments in set D were recorded from within the epileptogenic zone, and those in set $\mathrm{C}$ from the hippocampal formation of the opposite hemisphere of the brain. While sets C and D con-

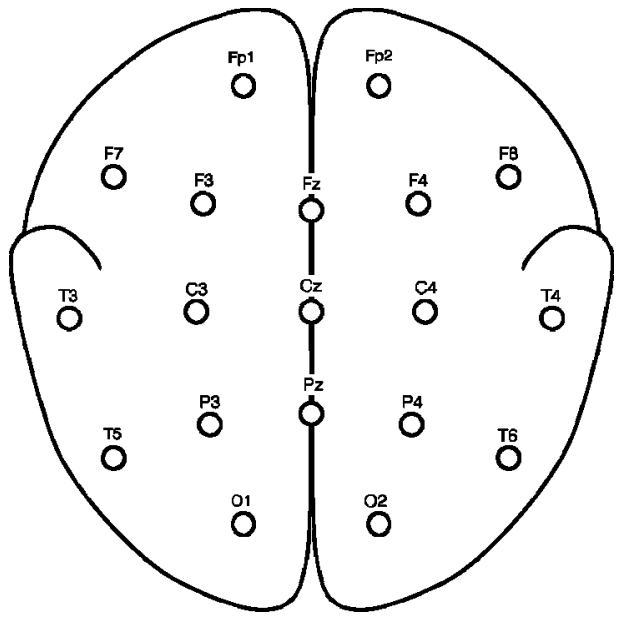

FIG. 1. Scheme of the locations of surface electrodes according to the international 10-20 system. Names of the electrode positions are derived from their anatomical locations. Segments of sets A and B were taken from all depicted electrodes.

tained only activity measured during seizure free intervals, set $\mathrm{E}$ only contained seizure activity. Here segments were selected from all recording sites exhibiting ictal activity.

All EEG signals were recorded with the same 128channel amplifier system, using an average common reference [omitting electrodes containing pathological activity $(\mathrm{C}$, $\mathrm{D}$, and $\mathrm{E}$ ) or strong eye movement artifacts (A and B)]. After 12 bit analog-to-digital conversion, the data were written continuously onto the disk of a data acquisition computer system at a sampling rate of $173.61 \mathrm{~Hz}$. Band-pass filter settings were $0.53-40 \mathrm{~Hz}$ (12 dB/oct.). Exemplary EEGs are depicted in Fig. 3.

\section{B. Steps of analysis}

\section{Surrogate time series}

For each time series $s=39$ surrogate time series were generated using the technique of Schreiber and Schmitz [47]. This iterative amplitude adjusting scheme results in surrogates that consist of the original sample values and have power spectra "practically indistinguishable" [47] from those of the original time series. The underlying null hypoth-
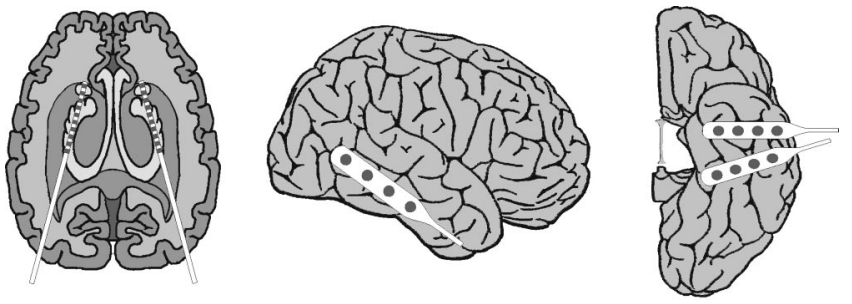

FIG. 2. Scheme of intracranial electrodes implanted for presurgical evaluation of epilepsy patients. Depth electrodes were implanted symmetrically into the hippocampal formations (top). Segments of sets $\mathrm{C}$ and $\mathrm{D}$ were taken from all contacts of the respective depth electrode. Strip electrodes were implanted onto the lateral and basal regions (middle and bottom) of the neocortex. Segments of set E were taken from contacts of all depicted electrodes. 


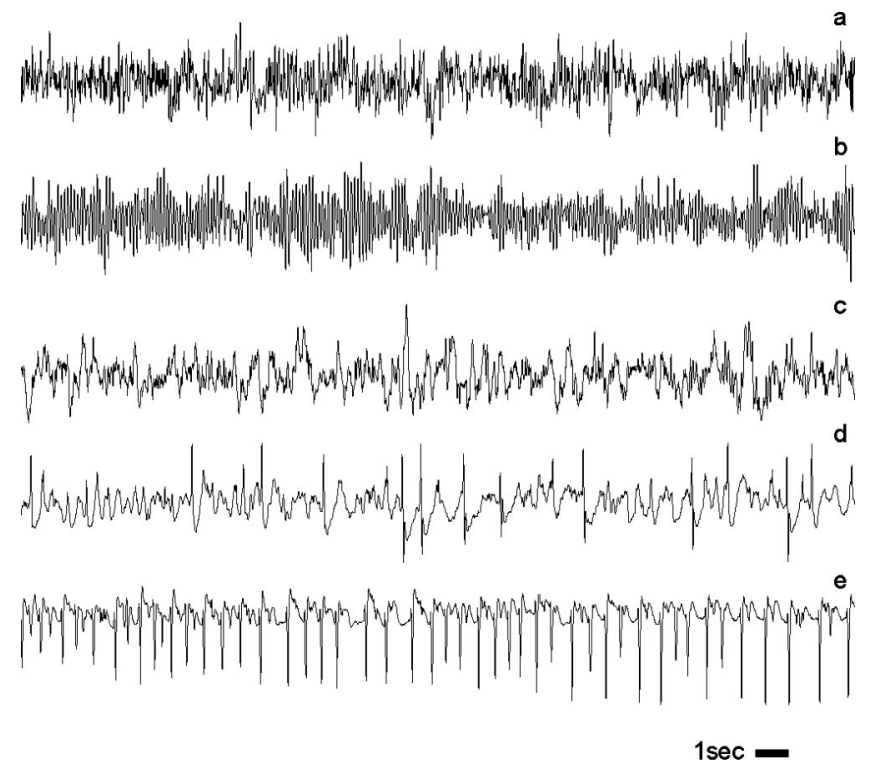

FIG. 3. Exemplary EEG time series from each of the five sets. From top to bottom: set A to set E (denoted EEG-a to EEG-e). Amplitudes of surface EEG recordings are typically in the order of some $\mu \mathrm{V}$. For intracranial EEG recordings amplitudes range around some $100 \mu \mathrm{V}$. For seizure activity these voltages can exceed $1000 \mu \mathrm{V}$.

esis is that the time series is compatible with a Gaussian linear stochastic and stationary process measured by a static and monotonic, possibly nonlinear, function. Since discontinuities between the end and beginning of a time series are known to cause spurious spectral frequency components, segments of 4396 samples were at first cut out of the recordings. Within these longer intervals, the beginning of each of the final segments of $N=4096$ samples was then chosen in such a way that the amplitude difference of the last and first data points was within the range of amplitude differences of consecutive data points, and the slopes at the end and beginning of the time series had the same sign. This algorithm avoids the use of window functions for a calculation of the power spectrum. A comparable technique was applied in Ref. [18].

\section{Weak stationarity criterion}

The probative force of a rejection of the surrogates' null hypothesis for deterministic and/or low-dimensional structure is limited, since stationarity is included in this null hypothesis. On the other hand, this particular property allows one to use surrogates in a test for nonstationarity. Here we propose such a test as a preanalysis step that eventually reduces the probability of rejections of the null hypothesis due to nonstationarities.

EEG-time series and respective surrogates were each divided into $n=16$ nonoverlapping subsegments (length $N^{*}$ =256). For each subsegment $(j=1, \ldots, n)$ the average deviation of amplitudes

$$
m_{j}^{x}=\frac{1}{N^{*}} \sum_{i=1}^{N^{*}}\left|x_{i}-\bar{x}\right|
$$

(where $\bar{x}$ denotes the mean of the respective subsegment) and the center frequency

$$
m_{j}^{\omega}=\frac{2}{N^{*}} \sum_{i=1}^{N^{* / 2}} \frac{\omega_{i} S\left(\omega_{i}\right)}{\overline{S\left(\omega_{i}\right)}}
$$

(where $S$ denotes the amplitude of the Fourier transform of the respective subsegment) were calculated. The fluctuation of these properties among subsegments was quantified using the average deviations

$$
\begin{aligned}
F^{x} & =\frac{1}{n} \sum_{j=1}^{n}\left|m_{j}^{x}-\overline{m^{x}}\right|, \\
F^{\omega} & =\frac{1}{n} \sum_{j=1}^{n}\left|m_{j}^{\omega}-\overline{m^{\omega}}\right|,
\end{aligned}
$$

where the overbar denotes mean of all 16 subsegments. As an inclusion criterion, both $F^{\omega}$ and $F^{x}$ of the original time series were required to range within the distribution calculated from the respective surrogate ensemble, i.e., both values must neither exceed the maximum nor fall below the minimum of the surrogate distribution.

\section{Estimate of an effective correlation dimension}

Using delay coordinates $\mathbf{x}_{i}=\left(x_{i}, x_{i+\tau}, \ldots, x_{i+(m-1) \tau}\right)$ [46] and the correlation sum

$$
C(\varepsilon, N)=\frac{2}{(N-T)(N-T-1)} \sum_{i=0}^{N-1} \sum_{j=i+T}^{N-1} \Theta\left(\varepsilon-\left\|\mathbf{x}_{i}-\mathbf{x}_{j}\right\|\right)
$$

[where $\Theta$ is the Heaviside step function, $\Theta(a)=0 \forall a \leqslant 0$ and $\Theta(a)=1 \forall a>0]$ the correlation dimension $D_{2}$ is defined [45] by

$$
D_{2}=\lim _{N \rightarrow \infty} \lim _{\varepsilon \rightarrow 0} d(\varepsilon, N),
$$

where

$$
d(\varepsilon, N)=\frac{\partial \ln C(\varepsilon, N)}{\partial \ln \varepsilon}
$$

From these definitions it follows that a true correlation dimension cannot be calculated from time series of finite length and limited accuracy. An estimation of an effective correlation dimension, however, can be obtained for those time series where a quasiscaling behavior of $d(\varepsilon, N)$ is found at least for a limited range of the hypersphere radius $\varepsilon$ [50]. The applied steps of analysis and the choice of parameters follow Ref. [36], and use the algorithm described in Ref. [51]. With a fixed time delay ( $\tau=1$ sampling time), $d(\varepsilon, N)$ was calculated for a range of embedding dimensions ( $m$ $=1, \ldots, 25)$ using the maximum norm and applying a Theiler window [52] ( $T=5$ sampling times). The range of $\varepsilon$ 
was chosen to match the resolution of the analog-to-digital converter, and divided into 128 intervals. A "quasiscaling region" $\left[\varepsilon_{l}, \varepsilon_{u}\right]$ is defined by

$$
\begin{aligned}
& \varepsilon_{u}=\max \left\{\varepsilon \mid d(\varepsilon, N)_{\mid m=1}>0.975\right\}, \\
\varepsilon_{l}= & \min \left\{\varepsilon|| d\left(\varepsilon_{u}, N\right)_{\mid m=25}-d(\varepsilon, N)_{\mid m=25} \mid\right. \\
\leqslant & \left.0.05 d(\varepsilon, N)_{\mid m=25}\right\} .
\end{aligned}
$$

If $\varepsilon_{u}$ and $\varepsilon_{l}$ existed, and the number $N_{r}$ of $\varepsilon$ values in $\left[\varepsilon_{l}, \varepsilon_{u}\right]$ was greater than or equal to 5 , the estimate

$$
D_{2, e f f}=\frac{1}{N_{r}} \sum_{\varepsilon=\varepsilon_{l}}^{\varepsilon_{u}} d(\varepsilon, N)_{\mid m=25}
$$

was computed. If no quasiscaling existed or if $D_{2, e f f} \geqslant 7.2$ $\approx 2 \log N$ (cf. Ref. [53]), $D_{2, \text { eff }}$ was set to an arbitrary value of $D_{u}=10$.

\section{Nonlinear prediction error}

For each reference point $\mathbf{x}_{i}(i=1, \ldots, N-m \tau)$ in a reconstructed state space (embedding dimension $m=6$ and time delay $\tau=8$ sampling times) a fixed number $(k=5)$ of nearest neighbors $\left\{\mathbf{x}_{j}\right\}_{j=1, \ldots, k}$ was used to perform an $H$-step prediction:

$$
\widetilde{\mathbf{x}_{i+H}}=\frac{1}{k} \sum_{j=1}^{k} \mathbf{x}_{j+H}
$$

The difference between the actual $\mathbf{x}_{i+H}$ and predicted translation $\widetilde{\mathbf{x}_{i+H}}$ is the local prediction error

$$
\varepsilon_{i, \widetilde{\mathbf{x}_{i+H}}}=\left|\mathbf{x}_{i+H}-\widetilde{\mathbf{x}_{i+H}}\right| .
$$

The local prediction error for the mean of the time series $\bar{x}$ is

$$
\varepsilon_{i, \overline{\mathbf{x}}}=\left|\mathbf{x}_{i+H}-\overline{\mathbf{x}}\right|,
$$

where $\overline{\mathbf{x}}$ is an $m$-dimensional vector that carries the mean in each of its component. Finally the nonlinear prediction error $(P)$ is calculated from

$$
P=\frac{\mathcal{R}\left(\varepsilon_{i, \widetilde{\mathbf{x}_{i+H}}}\right)}{\mathcal{R}\left(\varepsilon_{i, \overline{\mathbf{x}}}\right)},
$$

where $\mathcal{R}$ indicates the root mean square. For a nearest neighbor search for every reference point $\mathbf{x}_{i}$, a Theiler window ( $T=25$ sampling times) was applied to its own trajectory segment and to neighboring trajectory segments: In a first step, vectors with indices $\{i-T, \ldots, i-1, i+1, \ldots, i+T\}$ were discarded. In a second step, out of a group of nearest neighbors passing the first step but closer to each other in time than $T$, only the one nearest to $\mathbf{x}_{i}$ was included. The second step is important to ensure that information for the $P$ is gathered from multiple adjacent trajectory segments rather than from only one trajectory segment (cf. Refs. [48,49]). The prediction horizon $H$ was set to 65 sampling times. The parameter values reported were obtained from preanalysis with regard to an optimum differentiation between nonlinear deterministic and linear stochastic model systems. In order to meet the requirements of the respective statistics, we chose all parameters independently for both $P$ and $D_{2, e f f}$.

\section{Levels of null hypothesis testing - Statistical methods}

All following steps of analysis were carried out for $P$ and $D_{2}, e f f$. In the following, $M$ stands for any of the two measures. The null hypothesis was tested on two levels: on one level for every individual EEG segment, and on a second level for each of the five sets of EEG segments. On the individual level the null was rejected if $M^{E E G}$ ranged outside the distribution calculated from all of its surrogates $\left\{M^{\operatorname{Sur}_{i=1, \ldots, s}}\right\}$. For a given set and measure, let $R_{\text {min }}$ be the number of rejections of the null hypothesis which were caused by the fact that $M^{E E G}$ was smaller than the minimum of $\left\{M^{S u r_{i=1, \ldots, s}}\right\}$. To rate a given number of rejections of the null hypothesis, the probability $p_{\min }$ to find $R_{\min }$ or less rejections on a set of $n=100$ time series just by chance was calculated as

$$
p_{\min }=\sum_{k=0}^{R_{\min }}\left(\begin{array}{l}
n \\
k
\end{array}\right)\left(\frac{1}{s+1}\right)^{k}\left(1-\frac{1}{s+1}\right)^{n-k} .
$$

In complete analogy, we calculated the probability $p_{\max }$ to find $R_{\max }$ or less rejections that were caused by the fact that $M^{E E G}$ exceeded the surrogates' maximum.

On the set level one surrogate per EEG segment was used. In accordance with Ref. [3], values of $\left\{M^{E E G}\right\}$ and $\left\{M^{S U R_{1}}\right\}$ were used as paired observations for a nonparametric Wilcoxon signed rank test. In order to ensure that the choice of $D_{u}$ did not influence results of the null hypothesis testing on the set level, the Wilcoxon test was carried out with different values of $D_{u}$.

\section{RESULTS}

Figure 4 depicts results of exemplary EEGs. Table I and Fig. 5 summarize the results of all EEG segments and sets. On the individual level, sets E and D exhibited highest and second highest $R_{\min }$ values for both $P$ and $D_{2, \text { eff }}$. For all sets, the use of $P$ led to higher $R_{\min }$ values than the use of $D_{2, e f f}$. This was particularly true for sets B and C, for which the strongest discordance between the two measures was found: while for $P$ both $R_{\min }$ values were significant, they were both nonsignificant for $D_{2, e f f}$. Concordance was found only for set A: here none of the two measures led to significant $R_{\min }$ values. None of the $R_{\max }$ values was significant. Segments with rejection of the null hypothesis for both $P$ and $D_{2, e f f}$ were found in sets D and E only.

For the vast majority of segments $D_{2, e f f}$ could not be calculated, and was therefore set to $D_{u}$ (cf. Table I). For sets $\mathrm{A}$ and $\mathrm{B}$, this was the case for all EEG segments and almost all surrogates. Consequently, the null hypothesis on the set level could not be tested using $D_{2, e f f}$. For set C, only a few segments resulted in $D_{2, e f f}<D_{u}$, whereas most segments re- 

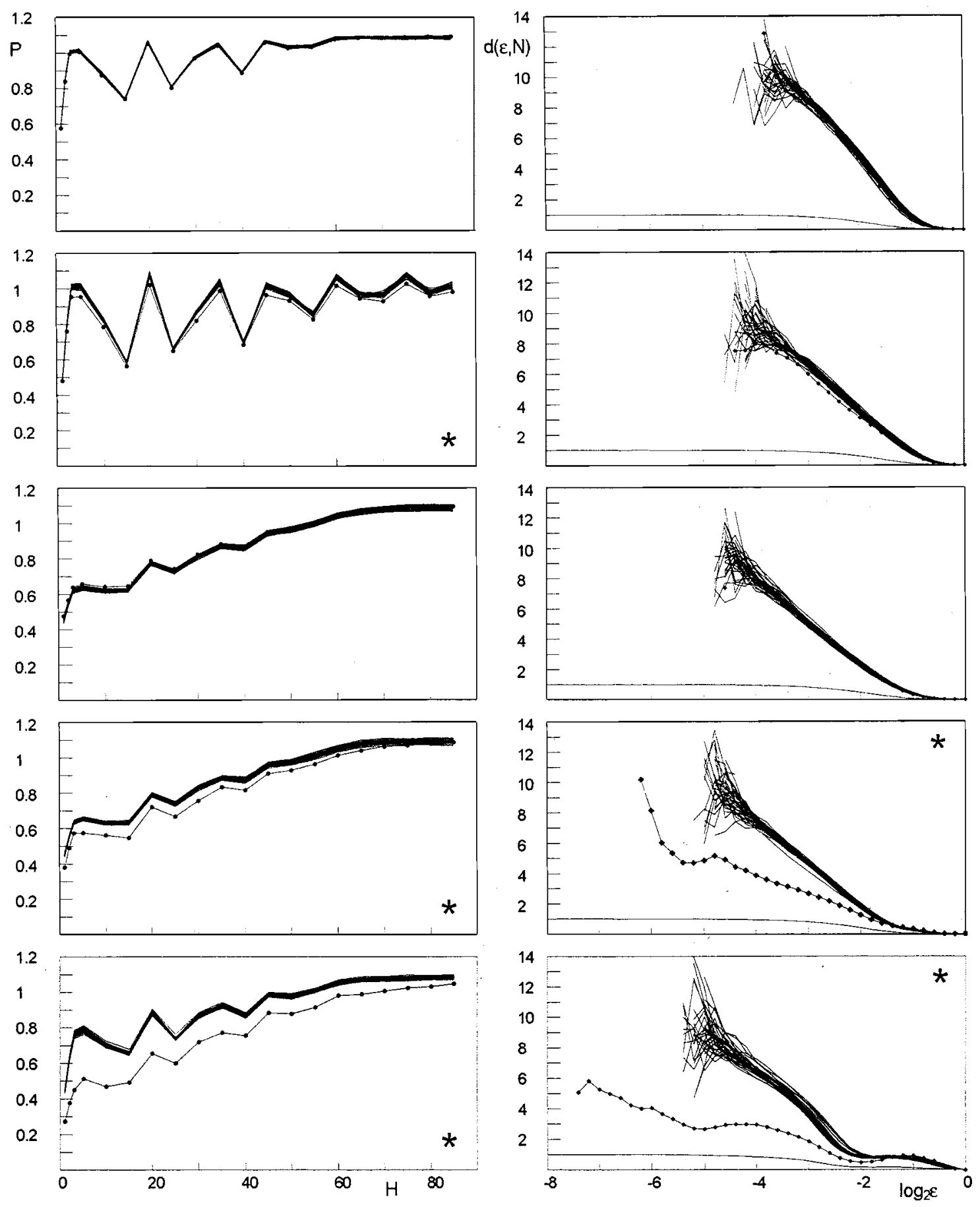

FIG. 4. Results for the exemplary EEG times series depicted in Fig. 3. From top to bottom: results for EEG-a to EEG-e. Left column: $P^{E E G}$ and $P^{S U R_{1, \ldots, s}}$ vs prediction horizon $H$. Circles mark $P^{E E G}$. Right column: $d(\varepsilon, N)^{E E G}$ and $d(\varepsilon, N)^{S U R_{1, \ldots, s}}$ vs the logarithm of the hypersphere radius $\varepsilon$ for an embedding dimension $m=25$. Diamonds mark $d(\varepsilon, N)^{E E G}$. In addition, $d(\varepsilon, N)^{E E G}$ for embedding dimension $m=1$ is given. Asterisks mark cases for which the null hypothesis was rejected. Note that for EEG-b there is a limited range of the hypersphere radius $\varepsilon$ for which $d(\varepsilon, N)^{E E G}$ was below the surrogates minimum. Hence the null hypothesis could be rejected. Neither the EEG nor the surrogate time series, however, resulted in a finite value of $D_{2, e f f}$, so that the null was not rejected for $D_{2, e f f}$. Only for examples EEG-d and EEG-e values of $D_{2, \text { eff }}$ could be estimated: 5.5 and 4.4, respectively.

sulted in $D_{2, e f f}^{E E G}=D_{2, e f f}^{S U R}=D_{u}$, and therefore entered the Wilcoxon test as binded observations. A noteworthy number of $D_{2, e f f}^{E E G}$ values was calculated only from EEG time series of sets D and E. These sets were the only ones that resulted in significant $p_{\text {Wilc }}$ values, so that the null hypothesis on the set

\section{level could be rejected.}

Using $P$ set A was the only one for which we obtained a nonsignificant $p_{\text {Wilc }}$ value. For all other sets the null hypothesis could be rejected on the set level. Although decreasing mean values of $P^{E E G}$ were found in the set order 
TABLE I. Results for $P$ and $D_{2, e f f}$ on the individual level (rows 1 and 2 and 6 and 7) and on the set level (rows 3 and 4 and 8 and 9). Values of $p_{\max }$ are not listed, since all were nonsignificant. Values in row 5 denote the number of segments for which a value of $D_{2, e f f}$ could be computed for the EEGs and the surrogates, respectively. The last row contains the number of segments for which the null was rejected for both measures. n.s.: values of $p>0.05$ are assumed to be nonsignificant. $x$ : no Wilcoxon test was carried out for sets A and B (see the body text).

\begin{tabular}{ccccccc}
\hline \hline & $\mathrm{A}$ & $\mathrm{B}$ & $\mathrm{C}$ & $\mathrm{D}$ & $\mathrm{E}$ \\
\hline \multirow{4}{*}{$P$} & $R_{\min } / R_{\max }$ & $4 / 1$ & $9 / 3$ & $14 / 3$ & $37 / 0$ & $89 / 0$ \\
& $p_{\min }$ & n.s. & $<0.001$ & $<0.001$ & $<0.001$ & $<0.001$ \\
$p_{\text {Wilc }}$ & n.s. & 0.004 & $<0.001$ & 0.001 & $<0.001$ \\
$Z_{\text {Wilc }}$ & -0.6 & -2.9 & -7.0 & -3.5 & -8.6 \\
& $D_{2, \text { eff }}^{E E G / S R_{1}}<D_{u}$ & $0 / 1$ & $0 / 1$ & $7 / 7$ & $27 / 9$ & $76 / 5$ \\
$D_{2, \text { eff }}$ & $R_{\min } / R_{\max }$ & $0 / 0$ & $0 / 0$ & $0 / 1$ & $17 / 2$ & $56 / 0$ \\
& $p_{\min }$ & n.s. & n.s. & n.s. & $<0.001$ & $<0.001$ \\
& $p_{\text {Wilc }}$ & $x$ & $x$ & n.s. & $<0.001$ & $<0.001$ \\
$P \& D_{2, \text { eff }}$ & $Z_{\text {Wilc }}$ & $x$ & $x$ & -1.0 & -3.7 & -7.6 \\
\hline \hline
\end{tabular}

A-C-B-D-E, smallest values were still found to be higher than 0.5 (cf. Fig. 5).

In summary, the null hypothesis of an underlying linear stochastic and stationary process, measured by a static and monotonic but possibly nonlinear measurement function as represented by the iterative amplitude adjusted surrogates, could be rejected for sets $\mathrm{D}$ and $\mathrm{E}$ for both $P$ and $D_{2, e f f}$. For sets B and C, only the use of $P$ led to a rejection, while these sets appeared to be compatible with the null for $D_{2, e f f}$. Set A was the only set which was compatible with the null hypothesis for both measures.

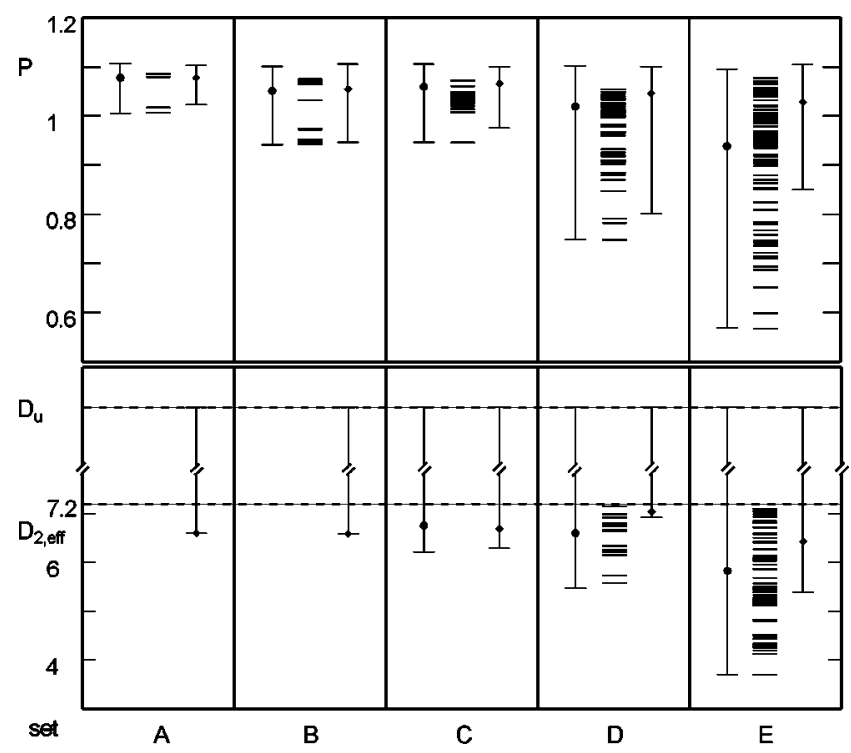

FIG. 5. Results for $P$ (upper panel) and $D_{2, \text { eff }}$ (lower panel) for sets A-E. For every measure and set, circles depict the mean value of $\left\{M^{E E G}\right\}$, and diamonds the mean value of $\left\{M^{S U R_{1}}\right\}$; bars on symbols are given by the ranges of these distributions. Vertical lines in between the symbols depict those values of $M^{E E G}$ for which the null hypothesis was rejected on the individual level by the fact that $M^{E E G}$ was smaller than the minimum of $\left\{M^{S U R_{1, \ldots, s}}\right\}$.

\section{DISCUSSION}

Our study showed clear differences in dynamical properties of brain electrical acitivity from different extracranial and intracranial recording regions and from different physiological and pathological brain states. Despite the assumed nonlinear deterministic nature of neuronal dynamics, a quasilinear stochastic and high-dimensional appearance of the EEG, as found for set A, consistent with results reported in Refs. $[4,9,15]$, might originate from both the huge number of neurons included in an EEG measurement and the complicated structure of the brain. Particularly for surface EEG recordings, a further blurring of possible dynamical structures in the EEG is caused by filter processes due to different conductivities of the skull and other intermediate tissue. Certain imposed constraints in dynamics, however, might demask or further strengthen nonlinear deterministic traits of neuronal dynamics. Results found with $P$ for set B suggest that the closing of eyes might represent the imposing of such a constraint in dynamics resulting in the well-known physiological alpha rhythm. For no segment of sets A and B, however, a conclusive indication of a finite dimension or even a low dimension could be obtained. Therefore, a differentiation of the conditions of eyes closed and eyes open, such as reported in Refs. [14,16], could not be obtained. Regarding these aspects, the results of our study are in agreement with other studies carried out on surface EEG recordings of healthy volunteers $[3,5,6,8,13,18]$, where indications of nonlinearity but not of an underlying low dimensional structure were found.

The strong indications of nonlinear deterministic structures found for set D were certainly often related to interictal epileptiform activity, as also reported in Refs. [28,29]. In some cases, however, NTSA measures might be capable of detecting more subtle dynamical manifestations of the disease epilepsy. This view was supported by a number of studies [26-28,30,34,41], all of which demonstrated a successful localization of the epileptogenic zone during seizure free in- 
tervals, and the anticipation of seizures by analysis of multichannel EEGs recorded from epilepsy patients [31,36-39]. The results of these studies, as well as the present one, suggest that the pathological epileptic process imposes certain constraints on neuronal dynamics. Even in the absence of seizure activity these constraints appear to be reflected by nonlinear deterministic traits in the EEG, as suggested by results found for set D. Most prominently, however, this phenomenon was observed during seizure activity (set E). This finding is in correspondence with previous studies on intra cranial [29] and extracranial [9,32] recordings of epileptic seizures. Theiler [32] even a priori assumed seizure activity to be "undoubtedly nonlinear."

When comparing extracranial and intracranial recording locations, it is important to note that the latter integrate potentials over a much smaller steradian, i.e., fewer neurons contribute to the measured potentials, which furthermore are less filtered as compared to extracranial recording locations. This might explain differences in results from sets A and C. An additional and not contradictory explanation might be given by the following. Set $\mathrm{C}$ was measured from brain regions which were proven to be nonepileptogenic but which may nevertheless participate in secondary, nonautonomous epileptic processes initiated by the epileptogenic zone.

The significant numbers of rejections $R_{\min }$ on the individual level allow two different interpretations. On the one hand, the sets could be assumed to have a certain distribution of some distinct dynamical property not included in the null hypothesis. Due to the limited sensitivity of the applied test statistics for this very property, the individual null will be rejected only for segments in one tail of this distribution. Differences of $R_{\min }$ values between sets could then be explained by different centers and widths of these distributions. Given the respective calculation parameters used in our study, both higher values and a higher intergroup variability of $R_{\min }$ were found for $P$ as compared to $D_{2, e f f}$. A comparison between Refs. [4], and [8], as well as data reported in Ref. [18] show analogous results. This could be interpreted as a higher degree of sensitivity of $P$ for indications of dynamical structures in EEG time series. The contrary conclusion, i.e., a lower level of specificity, cannot of course be ruled out.

On the other hand, the sets could consist of different subsets, each containing segments of different dynamical nature. This idea was followed in Ref. [8], where Stam et al. concluded that their "study underscores the heterogeneous nature $[\ldots]$ of the alpha rhythm from a dynamical point of view." This interpretation of different subsets would discour- age any further statistical analysis of the set level which assumes the sets to be homogeneous.

Doubtlessly, sets under investigation exhibited distinct linear properties described by power spectra and amplitude distributions. However, since these properties were shared by the surrogates, they cannot account for results given here. It is important, however, to point out that a rejection of the surrogates' null hypothesis is only a necessary but not sufficient criterion for nonlinearity. Surrogates that were used are stationary by construction. Therefore, even if a simple stationarity inclusion criterion was used to select the EEG segments, nonstationarity as a cause of "false" positive rejections cannot be excluded. Since this stationarity test is based on linear properties of the time series, it can neither be highly sensitive nor highly specific for nonstationarities of nonlinear dynamical systems. Nonlinear techniques calculated in a reconstructed state space such as the one proposed in Ref. [54] could help to overcome this shortcoming. Furthermore, Kugiumtzis [55] showed that although the iterative amplitude adjusted surrogates are more consistent in representing the given null hypothesis than older techniques [56], the remaining mismatch of linear correlations of the original time series can still be relevant, and can cause false rejections of the null. Since these and other shortcomings weaken the probative force of the applied method, we only use the term indication rather than evidence of nonlinear deterministic structures.

It has become a common point of view that values of an estimate of the correlation dimension calculated from an unknown dynamical system cannot be taken as a true estimate of a number of degrees of freedom. Particularly, our results give no indication of chaos in the underlying dynamical system brain. In a way this is to be expected, since a purely chaotic behavior of brain functions would consequently imply that our brains' behavior is changed dramatically by each and every arbitrary small input.

In accordance with other studies, our results show that an application of NTSA measures to EEG dynamics offers insights into the dynamical nature and variability of the system brain. As a feedback for NTSA, complicated properties of EEG dynamics can further motivate one to improve existing methods and develop new methods [57].

\section{ACKNOWLEDGMENTS}

We are grateful to Wieland Burr, Thomas Kreuz, Thomas Schreiber, and Guido Widman for useful discussions. This work was supported by the Deutsche Forschungsgemeinschaft.
[1] E. Ott, T. Sauer, and J. A. Yorke, Coping with Chaos (Wiley, New York, 1994).

[2] H. Kantz and T. Schreiber, Nonlinear Time Series Analysis (Cambridge University Press, Cambridge, England, 1997).

[3] S.A.R.B. Rombouts, R.W.M. Keunen, and C.J. Stam, Phys. Lett. A 202, 352 (1995).

[4] F.H. Lopes da Silva, J.P. Pijn, D. Velis, and P.C.G. Nijssen, Int.
J. Psychophysiol 26, 237 (1997).

[5] W.S. Pritchard, D.W. Duke, and K.K. Krieble, Psychophysiology 32, 486 (1995).

[6] M. Palus, Biol. Cybern. 75, 389 (1996).

[7] A.C.K. Soong and C.I.J.M. Stuart, Biol. Cybern. 62, 55 (1989).

[8] C.J. Stam, J.P.M. Pijn, P. Suffczynski, and F.H. Lopes da Silva, 
Clin. Neurophysiol. 110, 1801 (1999).

[9] H. Jing and M. Takigawa, Biol. Cybern. 83, 391 (2000).

[10] J. Jeong, M.S. Kim, and S.Y. Kim, Phys. Rev. E 60, 831 (1999).

[11] A. Babloyantz, J.M. Salazar, and G. Nicolis, Phys. Lett. A 111, 152 (1985).

[12] P. Achermann, R. Hartmann, A. Gunzinger, W. Guggenbuhl, and A.A. Borbely, Electroencephalogr. Clin. Neurophysiol. 90, 384 (1994).

[13] J. Fell, J. Röschke, and C. Schäffner, Biol. Cybern. 75, 85 (1996).

[14] C.J. Stam, T.C.A.M. van Woerkom, and W.S. Pritchard, Electroencephalogr. Clin. Neurophysiol. 99, 214 (1996).

[15] J. Theiler and P. Rapp, Electroencephalogr. Clin. Neurophysiol. 98, 213 (1996).

[16] B. Jelles, J.H. van Birgelen, J.P.J. Slaets, R.E.M. Hekster, E.J. Jonkman, and C.J. Stam, J. Clin. Neurophysiol. 110, 1159 (1999).

[17] P.E. Rapp, T.R. Bashore, J.A. Martinerie, A.M. Albano, I.D. Zimmermann, and A.I. Mees, Brain Topogr. 2, 99 (1989).

[18] C.L. Ehlers, J. Havstad, D. Prichard, and J. Theiler, J. Neurosci. 18, 7474 (1998).

[19] G. Widman, T. Schreiber, B. Rehberg, A. Hoeft, and C.E. Elger, Phys. Rev. E 62, 4898 (2000).

[20] J. Jeong, T.H. Chae, S.Y. Kim, and S.H. Han, J. Clin. Neurophysiol. 18, 58 (2001).

[21] V. Müller, W. Lutzenberger, F. Pulvermüller, B. Mohr, and N. Birbaumer, Exp. Brain Res. 137, 103 (2001).

[22] L. Pezard, R. Jech, and E. Ruzicka, Clin. Neurophysiol. 112, 38 (2001).

[23] C.J. Stam, T.C.A.M. van Woerkom, and R.W.M. Keunen, Biol. Cybern. 77, 247 (1997).

[24] J.-L. Nandrino, L. Pezard, J. Martinerie, F. El Massioui, B. Renault, R. Jouvent, J.-F. Allilaire, and D. Wildlöcher, NeuroReport 5, 528 (1994).

[25] P. Kirsch, C. Besthorn, S. Klein, J. Rindfleisch, and R. Olbrich, Brain Topogr 2, 187 (1990).

[26] K. Lehnertz and C.E. Elger, Electroencephalogr. Clin. Neurophysiol. 95, 108 (1995).

[27] B. Weber, K. Lehnertz, C.E. Elger, and H.G. Wieser, Epilepsia 39, 922 (1998).

[28] M.C. Casdagli, L.D. Iasemidis, R.S. Savit, R.L. Gilmore, S. Roper, and J.C. Sackellares, Electroencephalogr. Clin. Neurophysiol. 102, 98 (1997).

[29] J.P.M. Pijn, D.N. Velis, M.J. van der Heyden, J. DeGoedde, C. van Veelen, and F.H. Lopes da Silva, Brain Topogr 9, 249 (1997).

[30] R.G. Andrzejak, G. Widman, K. Lehnertz, C. Rieke, P. David, and C.E. Elger, Epilepsy Res. 44, 129 (2001).

[31] L.D. Iasemidis, P. Pardalos, J.C. Sackellares, and D.-S. Shiau, J. Combi. Optimization 5, 9 (2001).

[32] J. Theiler, Phys. Lett. A 196, 335 (1995).
[33] A. Babloyantz and A. Destexhe, Proc. Natl. Acad. Sci. U.S.A. 83, 3513 (1986).

[34] F. Mormann, K. Lehnertz, P. David, and C.E. Elger, Physica D 144, 358 (2000).

[35] G.W. Frank, T. Lookman, M.A.H. Nerenberg, C. Essex, J. Lemieux, and W. Blume, Physica D 46, 427 (1990).

[36] K. Lehnertz and C.E. Elger, Phys. Rev. Lett. 80, 5019 (1998).

[37] M. Le Van Quyen, J. Martinerie, V. Navarro, P. Boon, M. D'Have, C. Adam, B. Renault, F. Varela, and M. Baulac, Lancet 357, 183 (2001).

[38] J. Martinerie, C. Adam, M. Le Van Quyen, M. Baulac, S. Clemenceau, B. Renault, and F.J. Varela, Nat. Med. 4, 1173 (1998).

[39] K. Lehnertz, J. Arnhold, P. Grassberger, and C. E. Elger, Chaos in Brain? (World Scientific, Singapore, 2000).

[40] L.D. Iasemidis, J.C. Sackellares, H.P. Zaveri, and W.J. Williams, Brain Topogr. 2, 187 (1990).

[41] J. Arnhold, K. Lehnertz, P. Grassberger, and C.E. Elger, Physica D 134, 419 (1999).

[42] F.H. Lopes da Silva, Electroencephalogr. Clin. Neurophysiol. 79, 81 (1991).

[43] H. O. Lüders and I. Awad, in Epilepsy Surgery, edited by H. O. Lüders (Raven Press, New York, 1991), pp. 51-61.

[44] A. S. Weigend and N. A. Gershenfeld, Time Series Prediction: Forecasting the Future and Understanding the Past, Proceedings of the Santa Fe Institute Studies in the Science of Complexity Vol. XV (Addison -Wesley, Reading, 1993).

[45] P. Grassberger and I. Procaccia, Phys. Rev. Lett. 50, 346 (1983).

[46] F. Takens, in Dynamical Systems and Turbulence, edited by D. A. Rand and L.-S. Young, Lecture Notes in Mathematics Vol. 898 (Springer-Verlag, Berlin, 1980), p. 366-381.

[47] T. Schreiber and A. Schmitz, Phys. Rev. Lett. 77, 635 (1996).

[48] J. D. Farmer and J. J. Sidorowich, Exploiting Chaos to Predict the Future and Reduce Noise, in Evolution, Learning, and Cognition, edited by Y.-C. Lee (World Scientific, Singapore, 1988), pp. 277-330.

[49] J. McNames, Proceedings of the International Workshop on Advanced Black-Box Techniques for Nonlinear Modeling (Katholieke Universiteit, Leuven, 1998), pp. 112-128.

[50] P. Grassberger, T. Schreiber, and C. Schaffrath, Int. J. Bifurcation Chaos Appl. Sci. Eng. 1, 521 (1991).

[51] G. Widman, K. Lehnertz, P. Jansen, W. Meyer, W. Burr, and C.E. Elger, Physica D 121, 65 (1998).

[52] J. Theiler, Phys. Rev. A 34, 2427 (1986).

[53] J.-P. Eckmann and D. Ruelle, Physica D 56, 185 (1992).

[54] T. Schreiber, Phys. Rev. Lett. 78, 843 (1997).

[55] D. Kugiumtzis, Phys. Rev. E 60, 2808 (1999).

[56] J. Theiler, S. Eubank, A. Longtin, B. Galdrikian, and J.D. Farmer, Physica D 58, 77 (1992).

[57] EEG time series are available under http://www.meb.unibonn.de/epileptologie/science/physik/eegdata.html Contribution of further sets of EEG time series is encouraged. 